\title{
Observations of $\mathrm{H}_{2} \mathrm{O}$ maser and continuum emission in AFGL 2591
}

\author{
Miguel A. Trinidad, Salvador Curiel, Jorge Cantó \\ Instituto de Astronomía, (UNAM), Apdo. Postal 70-264, D.F. 04510, \\ México
}

José M. Torrelles

Institut d'Estudis Espacials de Catalunya (IEEC/CSIC) and Instituto de Ciencias del Espacio (CSIC), Edifici Nexus, C/ Gran Capità, 2-4, E-08034 Barcelona, Spain

Luis F. Rodríguez

Instituto de Astronomía, (UNAM), Apdo. Postal 3-72 (Xangari) 58089 Morelia, Michoacán, México

José F. Gómez

Laboratorio de Astrofísica Espacial y Física Fundamental (INTA), Apdo. Correos 50727, E-28080 Madrid, Spain

Paul T.P. Ho

Harvard-Smithsonian Center for Astrophysics, 60 Garden Street, Cambridge, MA 02138

\begin{abstract}
We report results of radio continuum $(1.3$ and $3.6 \mathrm{~cm})$ and $\mathrm{H}_{2} \mathrm{O}$ maser line observations, made with the Very Large Array (A configuration), toward the star-forming region AFGL 2591. We detected 85 maser spots toward this region, which are distributed in three main groups. Two of these groups spatially coincide with the radio continuum sources VLA 2 and VLA 3 . The maser spots associated with VLA 3 are distributed along a shell-like structure of $0^{\prime \prime} 01$ and nearly perpendicular to the CO bipolar outflow. We propose that VLA 3 is the center of the observed molecular flow in this region. Finally, we confirm that AFGL 2591 region is a cluster of B type stars, each one with its own optically thin H II region.
\end{abstract}

\section{Introduction}

AFGL 2591, located in the Cygnus X region, is a good laboratory to study some of the phenomena associated to star formation. It is a very luminous IR source $\left(9 \times 10^{4} \mathrm{~L}_{\odot}\right.$ at a distance of $2 \mathrm{Kpc}$, Lada et al. 1984), and is completely obscured at optical wavelengths. This infrared source is associated with a molecular outflow (Bally \& Lada 1983, Torrelles et al. 1983). Four radio continuum 
sources have been detected near AFGL 2591 (labeled from $\mathrm{n} 1$ to $\mathrm{n} 4$, Campbell 1984), but none of them were believed to coincide in position with this infrared source. A group of four maser spots was detected in the region by Tofani et al. (1995); three appeared clustered near the position of the K band IR source and the radio component $\mathrm{n} 3$ detected by Campbell (1984), while the other one was located near $\mathrm{n} 2$.

\section{Observations}

The observations were made with the VLA in its A configuration. We observed simultaneously $1.3 \mathrm{~cm}$ continuum and $\mathrm{H}_{2} \mathrm{O}$ maser emission, as well as $3.6 \mathrm{~cm}$ continuum emission. We used simultaneously two different bandwidths at 1.3 $\mathrm{cm}: 25 \mathrm{MHz}$ with seven channels for the continuum, and $3.125 \mathrm{MHz}$ with 63 channels for the line emission. The $\mathrm{H}_{2} \mathrm{O}$ line was centered at the frequency of the $6_{16} \rightarrow 5_{23}$ maser line (rest frequency $22235.080 \mathrm{MHz}$ ).

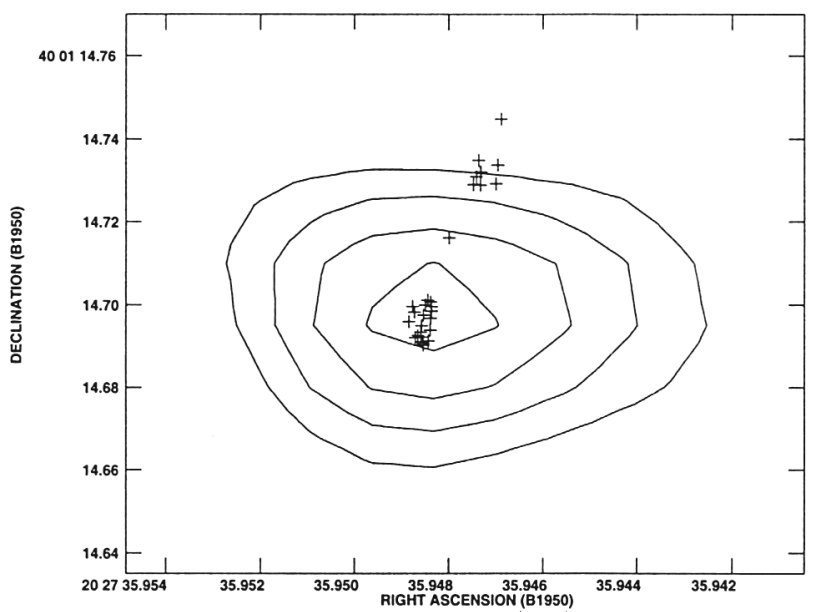

Figure 1. VLA-A continuum map of the radio source VLA 3 in the AFGL 2591 region at $1.3 \mathrm{~cm}$. Contours are $-4,-3,3,4,5,6,7$ times 0.19 mJy beam ${ }^{-1}$, the rms noise of the map (beam $=0$ ' 08 ). At this wavelength, only this continuum source was detected. Crosses indicate the position of the $\mathrm{H}_{2} \mathrm{O}$ maser spots associated with VLA 3.

\section{Results}

\subsection{Continuum Emission}

Three sources (VLA 1, VLA 2 and VLA 3) were detected in the region at 3.6 $\mathrm{cm}$. VLA 1 and VLA 2 appear resolved, being VLA 1 the most intense source in the region. The VLA 1 and VLA 2 flux densities are consistent with them being optically thin $\mathrm{H}$ II regions, which are photoionized by a zero-age main-sequence (ZAMS) B0.5 and B2 star, respectively. 
VLA 3 was detected at both wavelengths, 3.6 and $1.3 \mathrm{~cm}$ (Fig. 1). The calculated spectral index for VLA 3 in the wavelength range $[6-3.6 \mathrm{~cm}]$ is $\alpha=0.02$. Given the errors in the observed flux densities, this value is consistent with VLA 3 being an optically thin H II region ionized by a ZAMS B3 star. On the other hand, the millimeter emission of VLA 3 (van der Tak et al. 1999) is consistent with a spectral index of 2.3 , suggesting that the emission in this wavelength range is produced by heated dust. Therefore, VLA 3 could be an optically thin $\mathrm{H}$ II region surrounded by a dust envelope, probably a circumstellar disk.

\section{2. $\mathrm{H}_{2} \mathrm{O}$ Maser Emission}

We detected $85 \mathrm{H}_{2} \mathrm{O}$ maser spots in the region. The maser spots are mainly distributed in three clusters, one coincides spatially with VLA 3 (with 29 spots), another one appears to be associated with VLA 2 (22 maser spots) and the third one is located $\sim 0$ ' 5 north of VLA 3 (23 spots). Water maser emission is observed in the velocity range $V_{L S R}=-28$ to $+12.8 \mathrm{Km} \mathrm{s}^{-1}$, with the strongest component with intensity $S_{\nu}=70.3 \mathrm{Jy}$ and $V_{L S R}=-7.6 \mathrm{Km} \mathrm{s}^{-1}$.

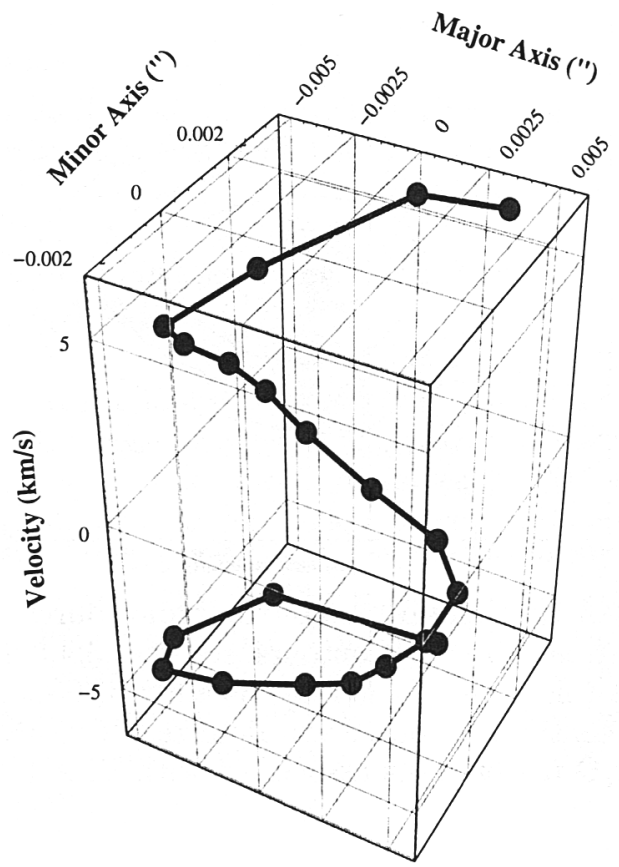

Figure 2. Three-dimensional position-velocity distribution of the subgroup of 20 maser spots associated with VLA 3. This figure shows that the maser spots have a continuous velocity gradient along the shell-like structure. The axes correspond to the relative position of the spots along the minor and major axis of the maser distribution with respect to their geometrical center. The $\mathrm{z}$ axis corresponds to the radial velocity of the maser spots after subtracting their systemic velocity of $-18 \mathrm{~km} \mathrm{~s}^{-1}$. 
A very nice result comes from the spatial-kinematical distribution of the water masers with respect to the VLA 3 peak continuum emission (Fig. 1). The $\mathrm{H}_{2} \mathrm{O}$ maser spots tend to be distributed in a strip of $\sim 0^{\prime}$.06 $(\sim 60$ AU at a distance of $1 \mathrm{Kpc}$ ) along the north-south direction, i.e., almost perpendicular to the CO bipolar outflow (aligned along the east-west direction; Mitchell, Maillard, \& Hasewaga 1991) and the $\mathrm{H}_{2}$ emission (Tamura \& Yamashita, 1992). The size of this structure and the spatial distribution of the maser spots suggest that these masers could be associated with a disk seen almost edge-on. This idea is also supported from the VLA 3 morphology. Although VLA 3 is not completely resolved, its radio emission appears is elongated in the direction of the bipolar molecular outflow (east-west). This scenario could suggest that VLA 3 is a radio jet located at the center of the outflow.

A subgroup of 20 of these maser spots, traces a shell-like structure of about 0. 01 ( 10 AU) nearly centered on VLA 3 and elongated almost in the northsouth direction. A very surprising result is obtained when we plot the spatialvelocity distribution of this subgroup of maser spots in three dimensions $(x, y, v)$. These maser spots show a continuous velocity gradient along the shell-like structure. Figure 2 shows that in three dimensions, the maser distribution forms a very well defined helical structure.

At present, we are analyzing possible physical situations that could give rise to this peculiar spatial-kinematical signature.

\section{References}

Bally J. \& Lada C. J. 1983, ApJ, 265, 824

Campbell B. 1984, ApJ, 287, 334

Lada C.J., Thronson H.A., Smith H.A., Schwartz, P.R., \& Glaccum, W. 1984, ApJ, 286, 302

Mitchell G.F., Maillard J., \& Hasewaga T.I. 1991, ApJ, 371, 342

Tamura, M., Gatley, I., Joyce, R.R., Ueno, M., Suto, H., \& Sekiguchi, M. 1991, ApJ, 378, 611

Tamura M. \& Yamashita T. 1992, ApJ, 391, 710

Tofani G., Felli M., Taylor G.B. and Hunter T.R. 1995, A\&AS, 112, 299

Torrelles J.M., Rodríguez L.F., Cantó J., Marcaide J., \& Gyulbudaghian A.L. 1983, RevMexAA, 8, 147

van der Tak F.F.S., van Dishoeck E.F., Evans II N.J., Bakker E.J., \& Blake G.A. 1999, ApJ, 522, 991 